\title{
Investigation of association of PPAR $\gamma$ gene Pro12Ala polymorphism with metabolic syndrome variables
}

\author{
A. AlSaleh, A. Soor, S. O'Dell and T. Sanders \\ Nutritional Sciences Division, King's College London, Franklin-Wilkins Building, 150 Stamford Street, \\ London SE1 9NH, UK
}

PPAR $\gamma$ is a transcription factor that is a member of the nuclear hormone receptor family ${ }^{(1)}$. PPAR $\gamma$ is believed to be a key player in metabolic syndromes, such as diabetes and obesity, and influences lipid metabolism by targeting specific genes. A coding SNP Pro12Ala $(\mathrm{C}>\mathrm{G}$ rs 1805192) in the PPAR $\gamma$ gene PPARG has been associated with insulin resistance and obesity-related traits of the metabolic syndrome, with the protective effect of the Ala $(G)$ allele being significant ${ }^{(2)}$. We genotyped Pro12Ala in 466 overweight/obese subjects who are at risk of the metabolic syndrome in the RISCK Study, a randomised parallel controlled trial, which aims to test the impact of specific dietary changes on cardiovascular risk factors associated with the metabolic syndrome ${ }^{(3)}$. We tested the association of genotype with mean quantitative metabolic phenotypes, LDL-C, HDL-C, TG, TC, adiponectin, BMI and insulin sensitivity. Baseline measurements were taken after following a reference diet containing $38 \%$ energy $(38 \% \mathrm{E})$ fat, $18 \% \mathrm{E}$ SFA, $10 \% \mathrm{E}$ MUFA, $6 \% \mathrm{E}$ PUFA for 1 month. The numbers of each genotype were in Hardy-Weinberg proportions as follows: $C / C 315, C / G 61, G / G 2$; $G$-allele frequency $=0.09$. A significant association was found with waist circumference in males after adjusting for age, whereby $G$-allele carriers had a lower waist circumference than $C C$ homozygotes $(P=0.020)$. $G$-allele carriers also had higher HDL-cholesterol compared to $C C$ homozygotes, although this association was insignificant after adjusting for age, gender and waist circumference $(P=0.079)$. No other significant associations with mean quantitative metabolic phenotypes were found after adjustment for age, gender and waist circumference. The Pro12Ala polymorphism seems to be associated with central obesity in males. The Ala $(G)$ allele may have protective effects in maintaining a healthy lipid profile. These findings may account for small inter-individual variation in fasting HDL-cholesterol concentration in obese/overweight subjects and may therefore modulate the risk of the metabolic syndrome.

1. Desvergne B \& Wahli W (1999) Peroxisome proliferator-activated receptors: nuclear control of metabolism. Endocr Rev 20, 649-688.

2. Ludovico O, Pellegrini F, Di Paola R et al. (2007) Heterogeneous effect of peroxisome proliferator-activated receptor $\gamma 2$ Ala12 variant on type 2 diabetes risk. Obesity 15, 1076-1081.

3. Jebb G, Frost G, Griffin J et al. (2007) The RISCK study: testing the impact of the amount and type of dietary fat and carbohydrate on metabolic risk. Nutr Bull 32, 154-156. 